Discussion This study suggests that a 'one-size fits all' approach to mattresses may not be appropriate and contrasting body types need different levels of support to improve overall spinal alignment. The use of simple anthropometric measurements could make the selection of the most appropriate mattress easier for the public.

\section{P003 RANDOMISED, PLACEBO-CONTROLLED STUDY OF SOLRIAMFETOL FOR EXCESSIVE DAYTIME SLEEPINESS IN NARCOLEPSY TYPES $1 / 2$}

1,2 Jed Black*, ${ }^{3}$ Yves Dauvilliers, ${ }^{4}$ Giuseppe Plazzi, ${ }^{5}$ Colin M Shapiro, ${ }^{6,7}$ Geert Mayer, ${ }^{8}$ Bruce C Corser, ${ }^{9}$ Helene A Emsellem, ${ }^{1}$ Dan Chen, ${ }^{1,10}$ Lawrence P Carter, ${ }^{11}$ Hao Wang, ${ }^{12}$ Michael J Thorpy. 'Jazz Pharmaceuticals, Palo Alto, USA; ${ }^{2}$ Stanford Center for Sleep Sciences and Medicine, Palo Alto, USA; ${ }^{3}$ Gui-de-Chauliac Hospital, CHU Montpellier, Montpellier, France; ${ }^{4}$ University of Bologna, Bologna, Italy; ${ }^{5}$ University of Toronto, Toronto, Canada; ${ }^{6}$ Hephata Klinik, Schwalmstadt, Germany; ${ }^{7}$ Philipps University Marburg, Marburg, Germany; ${ }^{8}$ Sleep Management Institute, Cincinnati, USA; ${ }^{9}$ The Center for Sleep and Wake Disorders, Chevy Chase, USA; ${ }^{10}$ University of Arkansas for Medical Sciences, Little Rock, USA; ${ }^{11}$ Kite Pharma, Inc., Santa Monica, USA, (previously from Jazz Pharmaceuticals, Palo Alto, CA, USA);

${ }^{12}$ Albert Einstein College of Medicine, Bronx, USA

\subsection{6/bmjresp-2019-bssconf.3}

Introduction Solriamfetol (formerly JZP-110), a dopamine and norepinephrine reuptake inhibitor, has been approved in the United States to improve wakefulness in adults with excessive daytime sleepiness (EDS) associated with narcolepsy (75-150 mg) or obstructive sleep apnoea (OSA; 37.5$150 \mathrm{mg}$ ). A Marketing Authorisation Application for these indications is under review with the European Medicines Agency. This phase 3 study assessed safety and efficacy of solriamfetol in participants with narcolepsy types 1 and 2 (NT1/2). ${ }^{1}$

Methods In this 12-week, double-blind, randomised, placebocontrolled study, participants with or without cataplexy were randomised to solriamfetol $75 \mathrm{mg}, 150 \mathrm{mg}, 300 \mathrm{mg}$, or placebo. Eligibility criteria: NT1/2 diagnosis; mean sleep latency $<25$ minutes on Maintenance of Wakefulness Test (MWT); Epworth Sleepiness Scale (ESS) score $\geq 10$. Exclusion criteria: medications that could affect EDS or cataplexy; night-time or variable shift work; other conditions causing EDS.

Results 236 participants received $\geq 1$ dose of solriamfetol (67.2\% female; 80.2\% white). Baseline MWT mean sleep latency: 7.5 minutes; baseline ESS score: 17.2. Solriamfetol significantly increased MWT sleep latency at week 12 $(\mathrm{P}<0.0001$ for $300 \mathrm{mg}$ and $150 \mathrm{mg})$; least squares (LS) mean change: 12.3 minutes for $300 \mathrm{mg}, 9.8$ for $150 \mathrm{mg}$, 4.7 for $75 \mathrm{mg}$, and 2.1 for placebo. Solriamfetol significantly decreased ESS scores at week $12(\mathrm{P}<0.0001150 \mathrm{mg}$ and $300 \mathrm{mg} ; \mathrm{P}<0.0575 \mathrm{mg}$ ). LS mean change in ESS: -6.4 for $300 \mathrm{mg},-5.4$ for $150 \mathrm{mg},-3.8$ for $75 \mathrm{mg}$, and -1.6 for placebo. Most common treatment-emergent adverse events (TEAEs; $\geq 5 \%$ ): headache, nausea, decreased appetite, nasopharyngitis, dry mouth, and anxiety. Discontinuations due to TEAEs were more frequent in solriamfetol $150 \mathrm{mg}$ and $300 \mathrm{mg}$ groups.

Discussion Solriamfetol improved wakefulness and reduced EDS in participants with NT1/2. Most AEs were mild to moderate.

Support Jazz Pharmaceuticals.
REFERENCE

1. Thorpy MJ, et al. Ann Neurol 2019;85(3):359-370.

\section{P004 SOLRIAMFETOL FOR EXCESSIVE DAYTIME SLEEPINESS IN OBSTRUCTIVE SLEEP APNOEA: A RANDOMISED CONTROLLED TRIAL}

${ }^{1}$ Paula K Schweitzer*, ${ }^{2,3}$ Geert Mayer, ${ }^{4,5}$ Russell Rosenberg, ${ }^{6,7}$ Gary K Zammit, ${ }^{8}$ Mark Gotfried, ${ }^{9}$ Dan Chen, ${ }^{9,10}$ Lawrence P Carter, ${ }^{11}$ Hao Wang, ${ }^{8,12}$ Jed Black, ${ }^{13}$ Atul Malhotra, ${ }^{14}$ Kingman P Strohl. 'Sleep Medicine and Research Center, St. Luke's Hospital, Chesterfield, USA; ${ }^{2}$ Hephata Klinik, Schwalmstadt, Germany; ${ }^{3}$ Philipps University Marburg, Marburg, Germany; ${ }^{4}$ NeuroTrialsResearch, Inc., Atlanta, USA; ${ }^{5}$ Atlanta School of Sleep Medicine, Atlanta, USA; ${ }^{6}$ Clinilabs Drug Development Corporation, New York, USA; ${ }^{7}$ Icahn School of Medicine at Mount Sinai, New York, USA; ${ }^{8}$ Pulmonary Associates, Phoenix, USA; ${ }^{9}$ Jazz Pharmaceuticals, Palo Alto, USA; ${ }^{10}$ University of Arkansas for Medical Sciences, Little Rock, USA; ${ }^{11}$ Kite Pharma, Inc., Santa Monica, USA, (previously from Jazz Pharmaceuticals, Palo Alto, CA, USA); ${ }^{12}$ Stanford Center for Sleep Sciences and Medicine, Palo Alto, USA; ${ }^{13}$ Division of Pulmonary, Critical Care and Sleep Medicine, University of California, San Diego, USA; ${ }^{14}$ Case Western Reserve University, Cleveland, USA

\subsection{6/bmjresp-2019-bssconf.4}

Introduction Obstructive sleep apnoea (OSA) is often associated with persistent excessive daytime sleepiness (EDS) despite sleep apnoea therapy. There are currently no approved treatments in the European Union for the treatment of EDS in this population. Solriamfetol (formerly JZP-110), a dopamine and norepinephrine reuptake inhibitor, has been approved in the United States to improve wakefulness in adults with EDS associated with narcolepsy $(75-150 \mathrm{mg})$ or OSA (37.5-150 mg). A Marketing Authorisation Application for these indications is under review with the European Medicines Agency. This study evaluated the efficacy and safety of solriamfetol for treatment of EDS in participants with OSA with current or prior sleep apnoea treatment. ${ }^{1}$

Methods In this double-blind, placebo-controlled, parallelgroup phase 3 trial, participants with OSA and associated EDS were randomly assigned to solriamfetol $37.5 \mathrm{mg}, 75 \mathrm{mg}, 150$ $\mathrm{mg}$, or $300 \mathrm{mg}$ or placebo for 12 weeks.

Results Of 476 randomised participants, 459 were included in the prespecified efficacy analyses. Co-primary endpoints (Maintenance of Wakefulness Test sleep latency, Epworth Sleepiness Scale score) were met at all solriamfetol doses $(\mathrm{P}<0.05)$, with dose-dependent effects observed at week 1 and maintained over the study duration. All doses except $37.5 \mathrm{mg}$ resulted in significantly higher percentages of participants reporting improvement on Patient Global Impression of Change (key secondary endpoint; $\mathrm{P}<0.05$ ). Adverse events (AEs) were reported in $47.9 \%$ of placebo- and $67.9 \%$ of solriamfetol-treated participants; 5 participants experienced serious AEs (2 [1.7\%] placebo, 3 [0.8\%] solriamfetol); none were deemed related to study drug. The most common AEs with solriamfetol were headache $(10.1 \%)$, nausea $(7.9 \%)$, decreased appetite $(7.6 \%)$, anxiety $(7.0 \%)$, and nasopharyngitis $(5.1 \%)$.

Discussion Solriamfetol significantly improved wakefulness and reduced sleepiness in participants with OSA and EDS. Most AEs were mild or moderate.

Support Jazz Pharmaceuticals.

\section{REFERENCE}

1. Schweitzer PK, et al. Am J Respir Crit Care Med 2019:199(11)1421-1431. 